Gontjes Kyle (Orcid ID: 0000-0001-8671-7990)

\title{
Contamination of Common Area and Rehabilitation Gym Environment with Multidrug- Resistant Organisms
}

Kyle J. Gontjes BS ${ }^{1,2}$; Kristen E. Gibson MPH${ }^{1}$; Bonnie Lansing LPN ${ }^{1}$; Marco Cassone MD $\mathrm{PhD}^{1}$; Lona Mody MD MSc ${ }^{1,3}$

${ }^{1}$ Division of Geriatric and Palliative Medicine, University of Michigan Medical School, Ann Arbor, MI, USA ${ }^{2}$ Department of Epidemiology, University of Michigan School of Public Health, Ann Arbor, MI, USA ${ }^{3}$ Geriatrics Research, Education and Clinical Center, Veterans Affairs Ann Arbor Healthcare System, Ann Arbor, MI, USA

Running Head: MDRO Contamination of NH Common Areas

\section{Corresponding Author:}

Lona Mody, MD, MSc, University of Michigan Medical School, Division of Geriatric and Palliative Medicine, 300 N Ingalls Rd, Room 905, Ann Arbor, MI 48109

Email: (lonamody@umich.edu)

Telephone: (734) 764-8942

Fax: (734) 936-2116

Twitter Handle: @LonaMody

This is the author manuscript accepted for publication and has undergone full peer review but has not been through the copyediting, typesetting, pagination and proofreading process, which may lead to differences between this version and the Version of Record. Please cite this article as doi: $10.1111 /$ jgs.16284

This article is protected by copyright. All rights reserved. 


\begin{abstract}
Objectives: To quantify the multidrug-resistant organism (MDRO) burden of high-touch common area and rehabilitation gym surfaces, and to assess microorganism transfer potential during rehabilitation sessions.
\end{abstract}

Design: Prospective study of environmental contamination.

Setting: Nursing Home.

Participants: Six Michigan Nursing Homes.

Measurements: Monthly samples from common area surfaces (e.g. living room), rehabilitation equipment, and rehabilitation personnel hands were screened for methicillin-resistant Staphylococcus aureus (MRSA), vancomycin-resistant enterococci (VRE) and resistant gramnegative bacilli (R-GNB). To assess microorganism transfer potential, we conducted an in-depth assessment of microorganism transfer during 10 rehabilitation sessions. Microorganism transfer was defined as the identification of a microorganism on a destination surface that was uncontaminated prior to the rehabilitation session. Additionally, patient frequency of common area usage was qualitatively assessed.

Results: We obtained 1338 common area specimens from 180 monthly facility visits, of which, 13.4\% (179/1338) were MDRO-positive: MRSA, 3.8\%; VRE, 5.8\%; R-GNB, 5.1\%. Sixty-four percent (116/180) of sampling visits had at least one MDRO-positive common area specimen. Within rehabilitation gyms, we obtained 521 equipment and 190 personnel hand specimens during 60 monthly visits. Of the equipment specimens collected, 7.7\% (40/521) were MDRO-

This article is protected by copyright. All rights reserved. 
positive: MRSA, 2.5\%; VRE, 4.0\%; R-GNB, 1.9\%. Of the 190 rehabilitation personnel hand specimens collected, 3.7\% (7/190) were MDRO-positive. Fifty-five percent (33/60) of rehabilitation gym visits had at least one MDRO-positive specimen. Microorganism transfer assessment during 10 rehabilitation sessions revealed 35 opportunities for transfer during which microorganism transfer occurred in 17.1\% (6/35) of opportunities.

Conclusion: Nursing home common areas and rehabilitation gyms are MDRO reservoirs that may contribute to the transmission of healthcare-associated pathogens. As NHs accommodate the increasing short-stay patient population, developing effective interventions that reduce MDRO transmission in the common area and rehabilitation gym environment should be considered an infection prevention priority.

Keywords: Multidrug-resistant organisms, environmental contamination, transmission, rehabilitation, nursing home.

This article is protected by copyright. All rights reserved. 


\section{INTRODUCTION}

As healthcare systems evolve to reduce hospital length of stay and expenditures, acute care patients are increasingly discharged to post-acute care facilities, predominantly for shortterm rehabilitation and subsequent discharge home ${ }^{1-3}$. Approximately $24 \%$ of such post-acute care patients are readmitted to acute care facilities within 30 days $^{4}$. This "revolving door" paradigm poses an increased threat of multidrug-resistant organism (MDRO) persistence and transmission within and beyond post-acute care facilities.

Colonization with MDROs predisposes patients to a heightened risk for adverse clinical outcomes, including infection ${ }^{5}$. Antimicrobial-resistant healthcare-associated infections are associated with increased complexity of care, rehospitalization risk, higher rates of morbidity and mortality, and considerable healthcare expenditures ${ }^{6-8}$. Post-acute care patients are at especially high risk for MDRO colonization and subsequent infection due to comorbidities, frailty, immunosenescence, presence of indwelling devices, prior hospitalization, and widespread exposure to antimicrobial therapy ${ }^{7,9}$. Moreover, treatment of antimicrobial-resistant infections in the older nursing home $(\mathrm{NH})$ population is challenging due to limited antimicrobial options, risk of drug toxicity, and polypharmacy interaction ${ }^{10,11}$. Henceforth, understanding the transmission dynamics and infection prevention concerns associated with MDROs in post-acute care facilities is critical to improving the safety of this patient population.

Environmental surfaces contribute to the persistence and transmission of MDROs within healthcare facilities ${ }^{12}$. Many common nosocomial pathogens can survive on environmental 
surfaces for weeks to months ${ }^{13}$. As reservoirs, environmental fomites have an increasingly evident role in direct and indirect transmission of nosocomial pathogens ${ }^{14-16}$. For example, we recently demonstrated that contamination of the patient room environment correlates with patient colonization in both acute and post-acute care settings ${ }^{17-19}$.

A growing body of evidence exists pertaining to the contribution of the proximal patient room environment ${ }^{18,20-22}$, medical equipment ${ }^{23}$, and nursing-associated surfaces ${ }^{24-26}$ to the persistence and transmission of MDROs in both acute and post-acute care settings. However, little is known about the contamination of high-touch surfaces in common areas. These shared environments are proposed to offer many opportunities for unintentional and unrecognized transmission $^{9,27}$. Environmental MDRO contamination and transmission dynamics associated with $\mathrm{NH}$ common areas is becoming increasingly compelling, as initial research has elucidated that environmental contamination of common areas is common ${ }^{28}$ and that associations exist between common area contamination and nursing facility methicillin-resistant Staphylococcus aureus (MRSA) burden ${ }^{29}$. However, despite their ubiquitous presence and emerging importance in NHs, there is an absence of literature on the environmental contamination and transmission potential associated with the $\mathrm{NH}$ rehabilitation gym environment. As social integration is critical to the enhancement of patient functionality, rehabilitation is central to the patient experience, and as patient MDRO colonization is common, these high-touch and high-traffic common areas may play an important role in the persistence and transmission of MDROs within NHs. 
In this study, we aimed to investigate the MDRO burden and transfer potential of hightouch common area and rehabilitation gym surfaces in the $\mathrm{NH}$ environment. Additionally, we sought to evaluate the importance of patient utilization of common areas, whilst examining differences in contamination between patient-used and nursing staff-used common area surfaces. In order to achieve these goals, we did the following: (1) surveyed patients on their common area usage, (2) quantified the MDRO burden of NH common areas, (3) quantified the MDRO burden of the rehabilitation gym environment (equipment surfaces and rehab personnel hands), and (4) conducted an in-depth assessment of microorganism transfer potential during ten rehabilitation sessions.

This article is protected by copyright. All rights reserved. 


\section{METHODS}

\section{Study Design}

We present data collected from a prospective, longitudinal cohort study conducted in 6 southeast Michigan NHs between January 2014 and August $2018^{30}$. The parent study was designed to characterize patient baseline MDRO colonization, new acquisition, and spontaneous losses throughout the duration of their stay. Patients were approached for enrollment regardless of patient characteristics, unless they were receiving end-of-life care. Microbial surveillance of patient colonization and patient room environment was conducted on patient enrollment, days 14, and monthly thereafter for a maximum of 6 months ${ }^{30}$.

In this study, we present data collected during microbial surveillance of NH common areas and in-depth sampling of the rehabilitation gym environment, alongside a pilot study that assessed microorganism transfer during a total of 10 rehabilitation sessions in the $\mathrm{NH}$ rehabilitation gym. From January 2014 to August 2016, the following high-touch common area surfaces, when available, were sampled monthly: shower room (handrail or shower chairs), rehabilitation gym (equipment), dining room (tabletop or chair), living room (tabletop or chair), hallway (soiled utility keypad, handrail, and nurses’ touch screen), and nurses’ station (countertop and patient charts). From November 2017 to August 2018, we sampled rehabilitation equipment and rehabilitation personnel (physical or occupational therapy) hands. Monthly samples from the rehabilitation gym environment were collected, when available, from the 
following surfaces: arm bike handle, pulley, stairs, mat, activity table, stationary bike handle, weights, chair handle, and parallel bars.

\section{Patient Data Collection}

Demographic data, such as age, gender, and race, and clinical data, such as antibiotic use, presence of infection(s), wound(s), or device use, were collected by trained research personnel upon patient enrollment and during follow-up visits. Patient usage of the following common areas was qualitatively assessed at enrollment: dining room, shower room, rehabilitation gym, and living room. Frequency of common area usage was defined categorically as either daily (5 days a week) or weekly ( $<5$ days a week).

\section{Microbiologic Methods}

Specimens were collected by trained research personnel using the BBL CultureSwab collection and transport system (Becton Dickinson, Cockeysville, MD) and enriched overnight at $36^{\circ} \mathrm{C}$ in brain heart infusion media. After enrichment, specimens were cultured onto mannitol salt agar, MacConkey agar, and bile-esculin agar with $6 \mu \mathrm{g} / \mathrm{mL}$ vancomycin for subsequent isolation. Staphylococcus aureus, vancomycin-resistant enterococci (VRE), and gram-negative bacilli (GNB) were identified using standard microbiology techniques ${ }^{30}$. Staphylococcus aureus was identified using StaphAurex Latex Agglutination Test (Remel, Lenexa, Kansas) and the catalase test. VRE was identified using the DrySlide PYR Kit (BD, Franklin Lakes, New Jersey). GNB was identified using the API-20E test system (bioMérieux, Genève, Switzerland). Antimicrobial resistance testing for S. aureus and GNB isolates was performed by disk diffusion 
using Clinical and Laboratory Standards Institute guidelines ${ }^{31}$. For S. aureus isolates, methicillin resistance was determined using $30 \mu \mathrm{g}$ cefoxitin disks ${ }^{31}$. Susceptible $S$. aureus isolates were classified as methicillin-susceptible Staphylococcus aureus (MSSA), while resistant isolates were classified as MRSA. For GNB isolates, resistance to one or more of the following antimicrobials constituted classification as resistant gram-negative bacilli (R-GNB): ceftazidime $(30 \mu \mathrm{g})$, ciprofloxacin $(5 \mu \mathrm{g})$, and imipenem $(10 \mu \mathrm{g})^{31}$.

\section{Assessment of Microorganism Transfer Potential During Patient Utilization of Rehabilitation Services}

To assess microorganism transfer during patient utilization of $\mathrm{NH}$ rehabilitation services, we followed four patients during a total of ten rehabilitation sessions between July 2018 and August 2018. Rehabilitation sessions were defined as the interaction of a patient with rehabilitation personnel with the intent to undergo physical therapy in the rehabilitation gym. Patient and rehabilitation personnel hand specimens were collected before initiation and after completion of the patient's entire rehabilitation session, while equipment surface specimens were collected before and after patient utilization (Figure 1). MDRO and non-MDRO burden was assessed using the aforementioned microbiology methods. Microorganism transfer was defined as the identification of microorganisms on destination surfaces that were not contaminated prior to source interaction ${ }^{32}$. Microorganism transfer directionality was classified as: (1) patient hands to rehabilitation equipment, (2) rehabilitation equipment to patient hands, or (3) unidentified rehabilitation environment reservoir to patient or rehabilitation personnel hands.

This article is protected by copyright. All rights reserved. 


\section{Statistical Analyses}

Patient demographics, clinical and common area usage data was assessed using proportions for categorical data and means for continuous data. Short-stay status was defined as patients with an expected duration of stay less than 90 days. MDRO burden of common areas was assessed using the following analysis strategies: (1) overall aggregate common area contamination data, (2) specimen-level data (aggregate MDRO burden of each common area surface sampled), and (3) visit-level data (aggregate MDRO burden of all common area surfaces sampled during that facility’s sampling visit). The MDRO burden of rehabilitation services was assessed using the aforementioned analysis strategies. Common area surfaces were dichotomized into primarily patient-used (shower room, rehabilitation gym, hallway handrails, living room, and dining room) and nursing staff-used (soiled utility room keypad, patient charts, nurses’ station counter, nurse touch screen) common areas through a priori reasoning. Univariate logistic regression was used to assess differences in MDRO contamination of primarily patient-used and nursing staff-used common area surfaces. Microorganism transfer during rehabilitation sessions were analyzed on surfaces with matched before and after specimens. Microorganism transfer potential was calculated as the number of identified transfer events divided by the total number of matched specimens. Analyses were performed using SAS, version 9.4 (SAS Institute, Inc., Cary, NC).

This article is protected by copyright. All rights reserved. 


\section{RESULTS}

\section{Patient Characteristics and Common Area Usage}

A total of 651 patients were enrolled in the parent study between January 2014 and August 2016. The average age was 74.7 years (range, 34-102; median, 76), 57.8\% were female, and $62.4 \%$ were white. At the time of admission, 92.5\% (602/651) of patients were expected to be short-stay residents. Patient demographics and enrollment statistics were described further elsewhere $^{30}$. Complete common area data was collected from 85.9\% (559/651) of enrolled patients. The most frequently used common areas were the shower room and rehabilitation gym, with 97.9\% (547/559) and 95.7\% (535/559) of enrolled patients reporting usage, respectively. Of these, 99.6\% (545/547) of shower room users reported weekly usage ( $<5$ days a week), while 99.4\% (532/535) of rehabilitation gym users reported daily usage (5 days a week). The dining room and living room were used less frequently, with $25.6 \%(143 / 559)$ and $18.8 \%(105 / 559)$ of enrolled patients reporting usage, respectively. Of these, 80.4\% (115/143) of dining room and $58.1 \%(61 / 105)$ of living room users reported daily usage.

\section{MDRO Burden of Nursing Home Common Areas}

During 180 monthly sampling visits, 1338 common area surfaces were swabbed from 54 total common areas. Of the 180 sampling visits, 116 (64.4\%) CA visits demonstrated the presence of at least one MDRO-positive common area specimen. Visit-level contamination of one or more common area with MRSA, VRE, and R-GNB was observed in 44 (24.4\%), 60

This article is protected by copyright. All rights reserved. 
(33.3\%), and 57 (31.7\%) of sampling visits, respectively. Additionally, of the 54 total common areas cultured during this study, 45 (83\%) were MDRO-positive at least once.

Of the 1338 common area specimens, 179 (13.4\%) were positive with at least one MDRO: 51 (3.8\%) with MRSA, 77 (5.8\%) with VRE, and 68 (5.1\%) with R-GNB. The most contaminated common area surfaces were the shower room (25.6\%), rehabilitation gym (21.4\%), hallway handrails (20.7\%), soiled utility room keypad (13.0\%), and the living room (11.1\%) (Table 1). Primarily patient-used common area surfaces were 3.1 times more likely to be contaminated with an MDRO than nursing-staff used common area surfaces (95\% confidence interval, 2.10-4.52; P-value, < 0.001).

\section{MDRO Burden of the Nursing Home Rehabilitation Gym}

To assess the MDRO burden of the NH rehabilitation gym, 711 targeted rehabilitation gym specimens (521 equipment and 190 personnel hand) were collected during 60 visits. Of the 60 rehabilitation gym sampling visits, 33 (55.0\%) demonstrated the presence of at least one MDRO-positive specimen. MRSA, VRE, and R-GNB were retrieved in 12 (20.0\%), 18 (30.0\%), and 13 (21.7\%) of the rehabilitation gym sampling visits, respectively. Of the 521 rehabilitation equipment specimens collected, 40 (7.7\%) were positive for at least one MDRO: 13 (2.5\%) with MRSA, 21 (4.0\%) with VRE, and 10 (1.9\%) with R-GNB. The most contaminated rehabilitation equipment was the arm bike handle (17.0\%), followed by the pulley (11.8\%), stairs (10.2\%), mat (8.5\%), and activity table (6.8\%) (Table 2). Of the 190 rehabilitation personnel hand specimens,

This article is protected by copyright. All rights reserved. 
7 (3.7\%) were MDRO-positive: 3 (1.6\%) with MRSA, 1 (0.5\%) with VRE, 3 (1.6\%) with RGNB.

\section{Microorganism Transfer Potential During Ten Rehabilitation Sessions}

In order to assess microorganism transfer potential associated with $\mathrm{NH}$ rehabilitation services, 4 patients were followed during a total of 10 rehabilitation session in two $\mathrm{NH}$ rehabilitation gyms for a total of 365 minutes (range, 13-54 minutes). The average participant age was 67.3 years (range, 62-74), 3 were female, and 3 were white. During these 10 rehabilitation sessions, 41 equipment, 20 patient hand, and 18 rehabilitation personnel hand specimens were collected. A total of 35 destination surfaces (10 patient hands, 7 rehabilitation personnel hands, and 18 equipment surfaces) had matching specimens collected (Figure 2). Half of 10 rehabilitation sessions demonstrated at least one microorganism transfer event. Of the 35 eligible destination surfaces during these 10 visits, $6(17.1 \%)$ demonstrated the acquisition of a novel microorganism (Figure 3).

This article is protected by copyright. All rights reserved. 


\section{DISCUSSION}

In this study, we conducted microbial surveillance in NH common areas to quantify their MDRO burden. Additionally, we assessed microorganism transfer potential through in-depth sampling during patient interaction with NH rehabilitation services. Our study demonstrates widespread MDRO burden in NH common areas, whilst providing evidence of pathogen transfer during patient and healthcare worker interaction within the rehabilitation gym environment. Our results further support the emerging infection prevention concern that high-touch surfaces located outside the patient room environment may contribute to the persistence and transmission of MDROs within post-acute care facilities ${ }^{9,27}$.

This study identified MDRO contamination in a significant proportion of specimens collected from common areas, whilst describing differences in MDRO burden among several high-touch common area and rehabilitation gym environment surfaces. These observations are supported by a limited number of studies. A study that conducted environmental sampling of high-touch common areas during a decolonization trial in 28 California NHs, reported that 50\% of common area specimens were contaminated with an $\mathrm{MDRO}^{28}$. In another study in 10 California NHs, Murphy et al. cultured the same ten common area objects during 5 separate visits and reported that $16 \%$ were MRSA-positive ${ }^{29}$. While the authors acknowledge that different microbiological techniques were used, our study reports markedly lower common area contamination than the two aforementioned studies, with MRSA and VRE identified on 3.8\% and $5.8 \%$ of common area specimens, respectively. While these aforementioned studies have 
initiated concern about environmental contamination of $\mathrm{NH}$ common areas, our study provides a broader account of the widespread contamination of NHs through our extensive surveillance of a diverse collection of both patient and nursing-associated common areas; and its assessment of common area contamination with R-GNB.

Additionally, we report widespread usage of $\mathrm{NH}$ common areas, with virtually all patients using the rehabilitation gym and shower room. Recent literature demonstrated that resident-staff, resident-environment, and staff-environment contact is frequent in $\mathrm{NH}$ common $\operatorname{areas}^{33}$. In particular, therapy-associated common areas were frequent sources of interaction for patients and nursing staff, alike ${ }^{33}$. Most notably, our findings demonstrated that primarily patient-used common areas (e.g. individual rehabilitation gym equipment) were more contaminated than common area surfaces used primarily by NH frontline staff (e.g. nurses' station). As patient social interaction is encouraged, these common areas could contribute to the persistence of MDROs by serving as environmental reservoirs.

Our finding that patient-used common areas are more likely to be contaminated with MDROs than nursing staff-used common areas is particularly intriguing. Studies now show that patient hands are more likely to be contaminated than healthcare provider hands ${ }^{17,34,35}$. As patient hand contamination with MDROs is higher than healthcare provider hand contamination with MDROs, these findings point to the role of patient hands in MDRO transmission. Furthermore, patient hands are markedly easy to culture, implicated as vehicles of transmission, and are therefore more likely to be informative when considering patient mobility outside of their 
rooms ${ }^{19,34}$. Our assessment of microorganism transfer potential provides evidence of patient seeding of environmental surfaces and subsequent acquisition potential for both patients and healthcare providers. These results further support the growing evidence of the transfer of microorganisms between patients, healthcare providers, and their environment during the provision of patient care ${ }^{32}$. Future studies should assess the role of pragmatic interventions, such as patient hand hygiene, in disrupting the chain of transmission during physical therapy in nursing homes.

Common area and rehabilitation gym environments present several challenging infection prevention concerns. First, transmission opportunities through the direct and indirect seeding of environmental surfaces, mediated by patient, staff, and visitor interaction, is frequent within these shared and high-trafficked environments. Additionally, patient interaction and the provision of care provides opportunities for direct MDRO transmission. Furthermore, $\mathrm{NH}$ environments are oftentimes designed with aspirations to promote an environment that encourages social interaction and patient autonomy. Infection prevention interventions, such as isolation precautions, the installation of wall-mounted alcohol-based hand rub dispensers, and the donning of personal protective equipment is oftentimes discouraged in an attempt to minimize perception of the $\mathrm{NH}$ as an institutionalized care setting ${ }^{27}$. Finally, attentiveness to environmental cleaning challenges is necessary in order to effectively reduce the MDRO burden and transmission potential associated with these environments. For instance, variations of common area cleaning practices have been reported to influence MRSA environmental burden ${ }^{29}$.

This article is protected by copyright. All rights reserved. 
Moreover, qualitative assessment of environmental cleaning practices at our study's 6 participating $\mathrm{NHs}$ identified heterogeneity in cleaning practices ${ }^{36}$. As the common area and $\mathrm{NH}$ rehabilitation environment pose infection prevention challenges, the development of appropriate policies, targeted procedures, and pragmatic interventions to address these concerns is advised ${ }^{37}$. Our study has several limitations. First, we sampled the common area and rehabilitation gyms monthly and thus could have missed transient contamination in between sampling visits. Second, as common area specimens were not differentiated by surface (e.g. tabletop or chair handle), this could have misrepresented the MDRO burden of each common area. This study's surface-differentiated sampling of the NH rehabilitation gym demonstrated this limitation. Third, the pilot assessment of microorganism transfer didn’t utilize genotyping to confirm isolate identity. We will be evaluating this question in our subsequent studies. Fourth, the sample size of the pilot qualitative assessment of MDRO transfer was limited to four enrolled patients over a duration of 10 total rehabilitation sessions. For more generalizable data, a larger and more representative patient sample is advised. Finally, this paper didn’t assess environmental cleaning and disinfection practices within the common area and rehabilitation gym environments. Future studies are warranted to assess the role of environmental cleaning practices in the reduction of pathogen burden on high-touch NH common area surfaces.

We note several strengths. First, this is one of the largest microbial surveillance studies of NH common area and rehabilitation gym surfaces - areas that are used by more than $95 \%$ of our burgeoning short-stay population. To the authors' knowledge, this study assesses several 
common area and rehabilitation equipment surfaces that were previously not reported as MDRO reservoirs. Second, our study's differential sampling of NH rehabilitation gym surfaces highlights the variable contamination of rehabilitation equipment surfaces, suggesting the potential for targeted infection prevention interventions. Third, this study assessed patient interaction with the surfaces located in the $\mathrm{NH}$ rehabilitation environment, demonstrating that the rehabilitation gym, an understudied infection prevention concern, is an MDRO reservoir with significant microorganism transfer potential.

In summary, this study supports the emerging understanding that environmental surfaces contribute to the persistence and transmission of MDROs within post-acute care facilities. This study's microbial surveillance of $\mathrm{NH}$ common areas identified widespread and frequent MDRO contamination, alongside transfer potential associated with the provision of physical therapy in the NH rehabilitation gym. Further research assessing MDRO transmission potential during patient interactions with the $\mathrm{NH}$ common area environment should be considered a priority. Additionally, assessment of facility-wide characteristics alongside patient-centric data could further elucidate the relationship between common area contamination and patient colonization in NHs.

This article is protected by copyright. All rights reserved. 


\section{ACKNOWLEDGMENTS}

The authors extend their gratitude to the patients, rehabilitation personnel, and the participating nursing homes. This work was supported by the National Institutes of Health (grant numbers RO1 AG041780 to L. M., and M. C.; K24 AG050685 to L. M); Pepper Center (pilot grant number AG024824 to M. C.); the Michigan Institute for Clinical and Health Research (grant number UL1TR002240 to L. M. and M. C.); and the National Institute on Aging (grant number P30 AG024824 to L. M.). L. M. is also supported by the Geriatrics Research, Education and Clinical Centers, Veterans Affairs Ann Arbor Healthcare System.

\section{Conflict of Interest}

The authors report no conflicts of interest.

\section{Author Contributions}

Study concept and design: K.J.G., L.M.

Acquisition of specimens and data: K.J.G., K.E.G., B.L.

Analysis and Interpretation of data: K.J.G, L.M.

Preparation of manuscript: All authors.

Sponsor's Role

None.

This article is protected by copyright. All rights reserved. 


\section{REFERENCES}

[1] Burke RE, Juarez-Colunga E, Levy C, Prochazka AV, Coleman EA, Ginde AA. Rise of Post-Acute Care Facilities as a Discharge Destination of US Hospitalizations. JAMA Internal Medicine. 2015;175: 295-296.

[2] Quinn CC, Port CL, Zimmerman S, et al. Short-Stay Nursing Home Rehabilitation Patients: Transitional Care Problems Pose Research Challenges. Journal of the American Geriatrics Society. 2008;56: 1940-1945.

[3] Werner RM, Konetzka RT. Trends in Post-Acute Care Use Among Medicare Beneficiaries: 2000 to 2015. JAMA. 2018;319: 1616-1617.

[4] Mor V, Intrator O, Feng Z, Grabowski DC. The revolving door of rehospitalization from skilled nursing facilities. Health Aff (Millwood). 2010;29: 57-64.

[5] Cassone M, Mody L. Colonization with Multi-Drug Resistant Organisms in Nursing Homes: Scope, Importance, and Management. Curr Geriatr Rep. 2015;4: 87-95.

[6] Strausbaugh LJ, Joseph CL. The Burden of Infection in Long-Term Care. Infection Control \& Hospital Epidemiology. 2000;21: 674-679.

[7] Montoya A, Mody L. Common infections in nursing homes: a review of current issues and challenges. Aging health. 2011;7: 889-899.

[8] Montoya A, Cassone M, Mody L. Infections in Nursing Homes: Epidemiology and Prevention Programs. Clinics in Geriatric Medicine. 2016;32: 585-607.

This article is protected by copyright. All rights reserved. 
[9] Dumyati G, Stone ND, Nace DA, Crnich CJ, Jump RLP. Challenges and Strategies for Prevention of Multidrug-Resistant Organism Transmission in Nursing Homes. Current Infectious Disease Reports. 2017;19: 18.

[10] Friedman ND, Temkin E, Carmeli Y. The negative impact of antibiotic resistance. Clinical Microbiology and Infection. 2016;22: 416-422.

[11] Giarratano A, Green SE, Nicolau DP. Review of antimicrobial use and considerations in the elderly population. Clinical interventions in aging. 2018;13: 657.

[12] Boyce JM. Environmental contamination makes an important contribution to hospital infection. Journal of Hospital Infection. 2007;65: 50-54.

[13] Kramer A, Schwebke I, Kampf G. How long do nosocomial pathogens persist on inanimate surfaces? A systematic review. BMC Infectious Diseases. 2006;6: 130.

[14] McDonald LC, Arduino M. Editorial commentary: climbing the evidentiary hierarchy for environmental infection control. Clin Infect Dis. 2013;56: 36-39.

[15] Suleyman G, Alangaden G, Bardossy AC. The Role of Environmental Contamination in the Transmission of Nosocomial Pathogens and Healthcare-Associated Infections. Current Infectious Disease Reports. 2018;20: 12.

[16] Otter JA, Yezli S, French GL. The Role Played by Contaminated Surfaces in the Transmission of Nosocomial Pathogens. Infection Control \& Hospital Epidemiology. 2011;32: 687-699.

This article is protected by copyright. All rights reserved. 
[17] Patel PK, Mantey J, Mody L. Patient Hand Colonization With MDROs Is Associated with Environmental Contamination in Post-Acute Care. Infection Control \& Hospital Epidemiology. 2017;38: 1110-1113.

[18] Cassone M, Mantey J, Perri MB, et al. Environmental Panels as a Proxy for Nursing Facility Patients With Methicillin-Resistant Staphylococcus aureus and Vancomycin-Resistant Enterococcus Colonization. Clin Infect Dis. 2018;67: 861-868.

[19] Mody L, Washer LL, Kaye KS, et al. Multidrug-resistant Organisms in Hospitals: What Is on Patient Hands and in Their Rooms? Clinical Infectious Diseases. 2019.

[20] Oie S, Hosokawa I, Kamiya A. Contamination of room door handles by methicillinsensitive/methicillin-resistant Staphylococcus aureus. Journal of Hospital Infection. 2002;51: 140-143.

[21] Trillis F, Eckstein EC, Budavich R, Pultz MJ, Donskey CJ. Contamination of Hospital Curtains With Healthcare-Associated Pathogens. Infection Control \& Hospital Epidemiology. 2008;29: 1074-1076.

[22] Huang SS, Datta R, Platt R. Risk of Acquiring Antibiotic-Resistant Bacteria From Prior Room Occupants. JAMA Internal Medicine. 2006;166: 1945-1951.

[23] Schabrun S, Chipchase L. Healthcare equipment as a source of nosocomial infection: a systematic review. Journal of Hospital Infection. 2006;63: 239-245.

This article is protected by copyright. All rights reserved. 
[24] Bures S, Fishbain JT, Uyehara CFT, Parker JM, Berg BW. Computer keyboards and faucet handles as reservoirs of nosocomial pathogens in the intensive care unit. American Journal of Infection Control. 2000;28: 465-471.

[25] Munoz-Price LS, Arheart KL, Mills JP, et al. Associations between bacterial contamination of health care workers' hands and contamination of white coats and scrubs. American Journal of Infection Control. 2012;40: e245-e248.

[26] Chen K-H, Chen L-R, Wang Y-K. Contamination of medical charts: an important source of potential infection in hospitals. PLoS One. 2014;9: e78512-e78512.

[27] Mody L, Bradley SF, Huang SS. Keeping the "home" in nursing home: implications for infection prevention. JAMA internal medicine. 2013;173: 853-854.

[28] McKinnell JA, Miller L, Singh RD, et al. When a Home is Not a Home: MultiDrugResistant Organism (MDRO) Colonization and Environmental Contamination in 28 Nursing Homes (NHs). Open Forum Infect Dis. 2017;4: S42-S43.

[29] Murphy CR, Eells SJ, Quan V, et al. Methicillin-resistant Staphylococcus aureus burden in nursing homes associated with environmental contamination of common areas. Journal of the American Geriatrics Society. 2012;60: 1012-1018.

[30] Mody L, Foxman B, Bradley S, et al. Longitudinal Assessment of Multidrug-Resistant Organisms in Newly Admitted Nursing Facility Patients: Implications for an Evolving Population. Clin Infect Dis. 2018;67: 837-844.

This article is protected by copyright. All rights reserved. 
[31] CLSI. Performance standards for antimicrobial susceptibility testing. 26th edn. Wayne, PA: Clinical and Laboratory Standards Institute, 2016.

[32] Wolfensberger A, Clack L, Kuster SP, et al. Transfer of pathogens to and from patients, healthcare providers, and medical devices during care activity—a systematic review and metaanalysis. Infection Control \& Hospital Epidemiology. 2018;39: 1093-1107.

[33] Pineles L, Perencevich EN, Roghmann M-C, et al. Frequency of nursing home resident contact with staff, other residents, and the environment outside resident rooms. Infection Control \& Hospital Epidemiology. 2019;40: 815-816.

[34] Cao J, Min L, Lansing B, Foxman B, Mody L. Multidrug-Resistant Organisms on Patients’ Hands: A Missed OpportunityMultidrug-Resistant Organisms on Patients’ HandsLetters. JAMA Internal Medicine. 2016;176: 705-706.

[35] Istenes N, Bingham J, Hazelett S, Fleming E, Kirk J. Patients' potential role in the transmission of health care-associated infections: Prevalence of contamination with bacterial pathogens and patient attitudes toward hand hygiene. American Journal of Infection Control. 2013;41: 793-798.

[36] Saeb A, Mody L, Gibson K. How are nursing homes cleaned? Results of a survey of 6 nursing homes in Southeast Michigan. American journal of infection control. 2017;45: e119e122.

This article is protected by copyright. All rights reserved. 
[37] Smith PW, Bennett G, Bradley S, et al. SHEA/APIC Guideline: Infection Prevention and Control In The Long-Term Care Facility. Infection Control \& Hospital Epidemiology. 2008;29: 785-814.

This article is protected by copyright. All rights reserved. 
Table 1. Multidrug-Resistant Organism (MDRO) Contamination of High-Touch Common Area Surfaces.

\begin{tabular}{|c|c|c|c|c|}
\hline Specimen & $\begin{array}{l}\text { MRSA+ } \\
\text { No. }(\%)\end{array}$ & $\begin{array}{l}\text { VRE+ } \\
\text { No. }(\%)\end{array}$ & $\begin{array}{l}\text { R-GNB+ } \\
\text { No. (\%) }\end{array}$ & $\begin{array}{l}\text { Any MDRO+ } \\
\text { No. (\%) }\end{array}$ \\
\hline $\begin{array}{l}\text { All Common Area Specimens } \\
(\mathrm{N}=1338)\end{array}$ & $51(3.8)$ & $77(5.8)$ & $68(5.1)$ & $179(13.4)$ \\
\hline $\begin{array}{l}\text { Primarily Patient-Used Common Areas } \\
(\mathrm{N}=796)^{\mathrm{a}}\end{array}$ & $43(5.4)$ & $61(7.7)$ & $52(6.5)$ & $143(18.0)$ \\
\hline Shower Room $(\mathrm{n}=156)$ & $7(4.5)$ & $20(12.8)$ & $19(12.2)$ & $40(25.6)$ \\
\hline Rehabilitation Gym $(\mathrm{n}=178)$ & $14(7.9)$ & $20(11.2)$ & $10(5.6)$ & $38(21.4)$ \\
\hline Hallway Handrails $(\mathrm{n}=179)$ & $14(7.8)$ & $10(5.6)$ & $13(7.3)$ & 37 (20.7) \\
\hline Living Room $(\mathrm{n}=117)$ & $2(1.7)$ & $5(4.3)$ & $6(5.1)$ & $13(11.1)$ \\
\hline Dining Room (n=166) & $6(3.6)$ & $6(3.6)$ & $4(2.4)$ & $15(9.0)$ \\
\hline $\begin{array}{l}\text { Nursing Staff-Used Common Areas } \\
(\mathrm{N}=542)\end{array}$ & $8(1.5)$ & $16(3.0)$ & $16(3.0)$ & 36 (6.6) \\
\hline Soiled Utility Room Keypad $(n=131)$ & $2(1.5)$ & $12(9.2)$ & $7(5.3)$ & $17(13.0)$ \\
\hline Patient Charts $(\mathrm{n}=71)$ & $0(0.0)$ & $3(4.2)$ & $3(4.2)$ & $6(8.5)$ \\
\hline Nurses’ Station Counter $(\mathrm{n}=173)$ & $5(2.9)$ & $0(0.0)$ & $5(2.9)$ & $10(5.8)$ \\
\hline Nurse Touch Screen (n=167) & $1(0.6)$ & $1(0.6)$ & $1(0.6)$ & $3(1.8)$ \\
\hline
\end{tabular}

${ }^{\mathrm{a}}$ Common area surfaces were stratified by patient and nursing staff usage pattern through a priori reasoning.

Abbreviations: MRSA, methicillin-resistant Staphylococcus aureus; VRE, vancomycin-resistant enterococci; R-GNB, resistant gram-negative bacilli; MDRO, multidrug-resistant organism.

This article is protected by copyright. All rights reserved. 
Table 2. Multidrug-Resistant Organism Contamination of Rehabilitation Gym Equipment and Rehabilitation Personnel Hand Specimens.

\begin{tabular}{|l|c|c|c|c|}
\hline \multicolumn{1}{|c|}{ Specimen } & $\begin{array}{c}\text { MRSA+ } \\
\text { No. (\%) }\end{array}$ & $\begin{array}{c}\text { VRE+ } \\
\text { No. (\%) }\end{array}$ & $\begin{array}{c}\text { R-GNB+ } \\
\text { No. (\%) }\end{array}$ & $\begin{array}{c}\text { Any MDRO+ } \\
\text { No. (\%) }\end{array}$ \\
\hline $\begin{array}{l}\text { Rehabilitation Personnel Hands } \\
\text { (N=190) }\end{array}$ & $\mathbf{3 ( 1 . 6 )}$ & $\mathbf{1 ~ ( 0 . 5 )}$ & $\mathbf{3 ( 1 . 6 )}$ & $\mathbf{7 ~ ( 3 . 7 )}$ \\
\hline $\begin{array}{l}\text { Rehabilitation Equipment } \\
\text { (N=521) }\end{array}$ & $\mathbf{1 3 ( 2 . 5 )}$ & $\mathbf{2 1 ( 4 . 0 )}$ & $\mathbf{1 0}(\mathbf{1 . 9})$ & $\mathbf{4 0}(\mathbf{7 . 7 )}$ \\
\hline Arm Bike Handle (n=53) & $4(7.6)$ & $3(5.7)$ & $4(7.6)$ & $9(17.0)$ \\
\hline Pulley (n=51) & $1(2.0)$ & $3(5.9)$ & $2(3.9)$ & $6(11.8)$ \\
\hline Stairs (n=59) & $1(1.7)$ & $3(5.1)$ & $2(3.4)$ & $6(10.2)$ \\
\hline Mat (n=59) & $2(3.4)$ & $2(3.4)$ & $2(3.4)$ & $5(8.5)$ \\
\hline Activity Table (n=59) & $2(3.4)$ & $2(3.4)$ & $0(0.0)$ & $4(6.8)$ \\
\hline Stationary Bike Handle (n=64) & $2(3.1)$ & $3(4.7)$ & $0(0.0)$ & $4(6.3)$ \\
\hline Weights (n=57) & $0(0.0)$ & $2(3.5)$ & $0(0.0)$ & $2(3.5)$ \\
\hline Chair Handle (n=59) & $1(1.7)$ & $1(1.7)$ & $0(0.0)$ & $2(3.4)$ \\
\hline Parallel Bars (n=60) & $0(0.0)$ & $2(3.3)$ & $0(0.0)$ & $2(3.3)$ \\
\hline
\end{tabular}

Abbreviations: MRSA, methicillin-resistant Staphylococcus aureus; VRE, vancomycin-resistant enterococci; R-GNB, resistant gram-negative bacilli; MDRO, multidrug-resistant organism.

This article is protected by copyright. All rights reserved. 


\section{FIGURE LEGENDS}

\section{Figure 1. Example of Microorganism Transfer During a Rehabilitation Session.}

Step 1: Patient and rehabilitation personnel (physical or occupational therapy) hand specimens were collected before initiation of the rehabilitation session. Step 2: Equipment surface swabs were collected before and after patient utilization. Step 3: Patient and rehabilitation personnel hand specimens were collected after the conclusion of the rehabilitation session. Microorganism transfer was defined as the identification of microorganisms on destination surfaces that were not contaminated prior to source interaction. Here, the patient successively interacts with the weights, walker, and stationary bike during their rehabilitation session. Transfer of methicillinsusceptible Staphylococcus aureus (MSSA) from the patient hands to the stationary bike handle was identified.

Figure 2. Assessment of Microorganism Transfer Potential During Ten Rehabilitation Sessions.

Abbreviations: MRSA, methicillin-resistant Staphylococcus aureus; MSSA, methicillinsusceptible $S$. aureus.

Figure 3. Six Microorganism Transfer Events Identified During Transfer Potential Assessment.

This article is protected by copyright. All rights reserved. 
Non-resistant Escherichia vulneris was classified as GNB. Abbreviations: MRSA, methicillinresistant Staphylococcus aureus; VRE, vancomycin-resistant enterococci; GNB, gram-negative bacilli.

This article is protected by copyright. All rights reserved. 
Step 1

(Before Rehab Visit)

Step 2

(During Rehab Visit)

Before Use: MSSAAfter Use: MSSA-

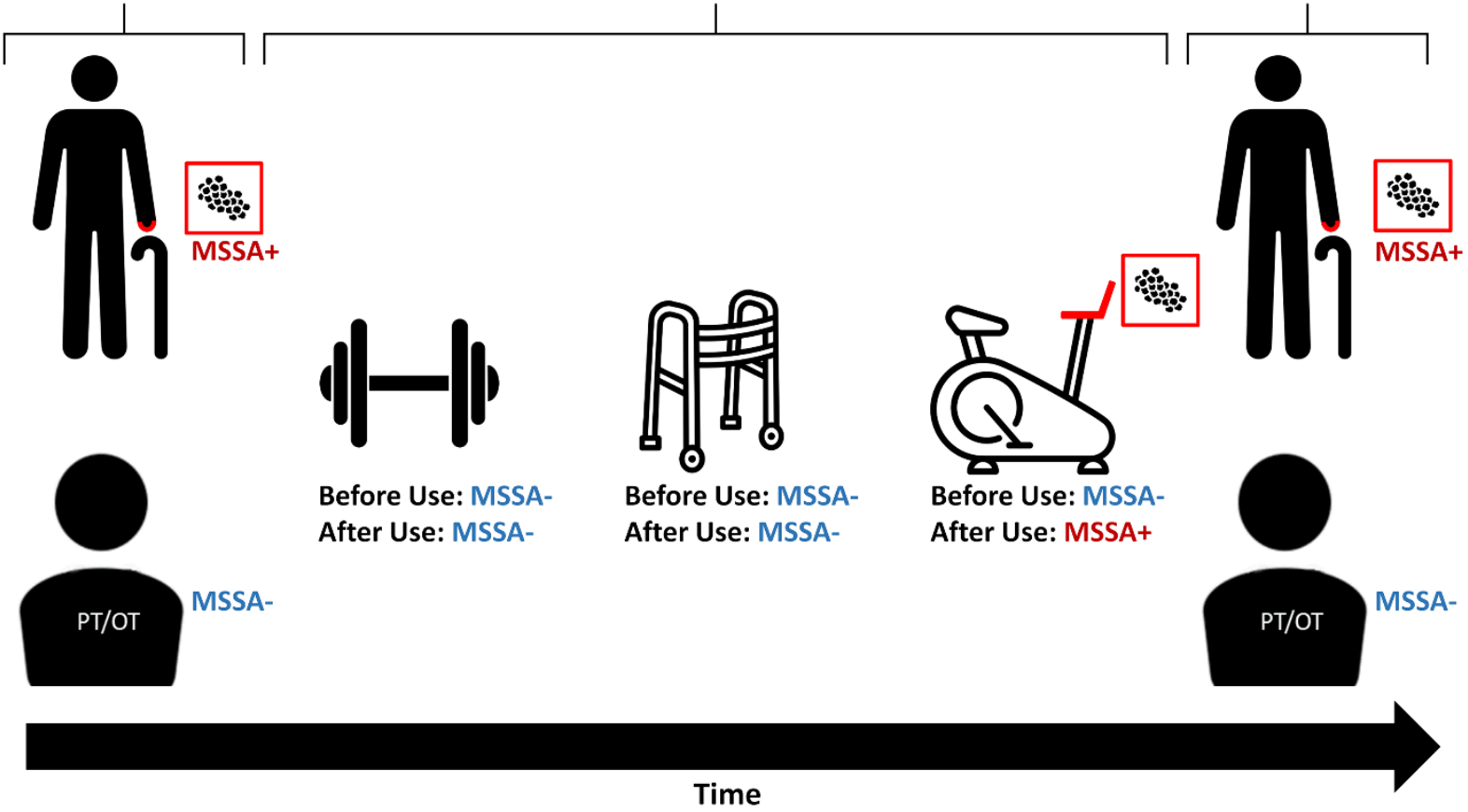

Before Use: MSSAAfter Use: MSSA-

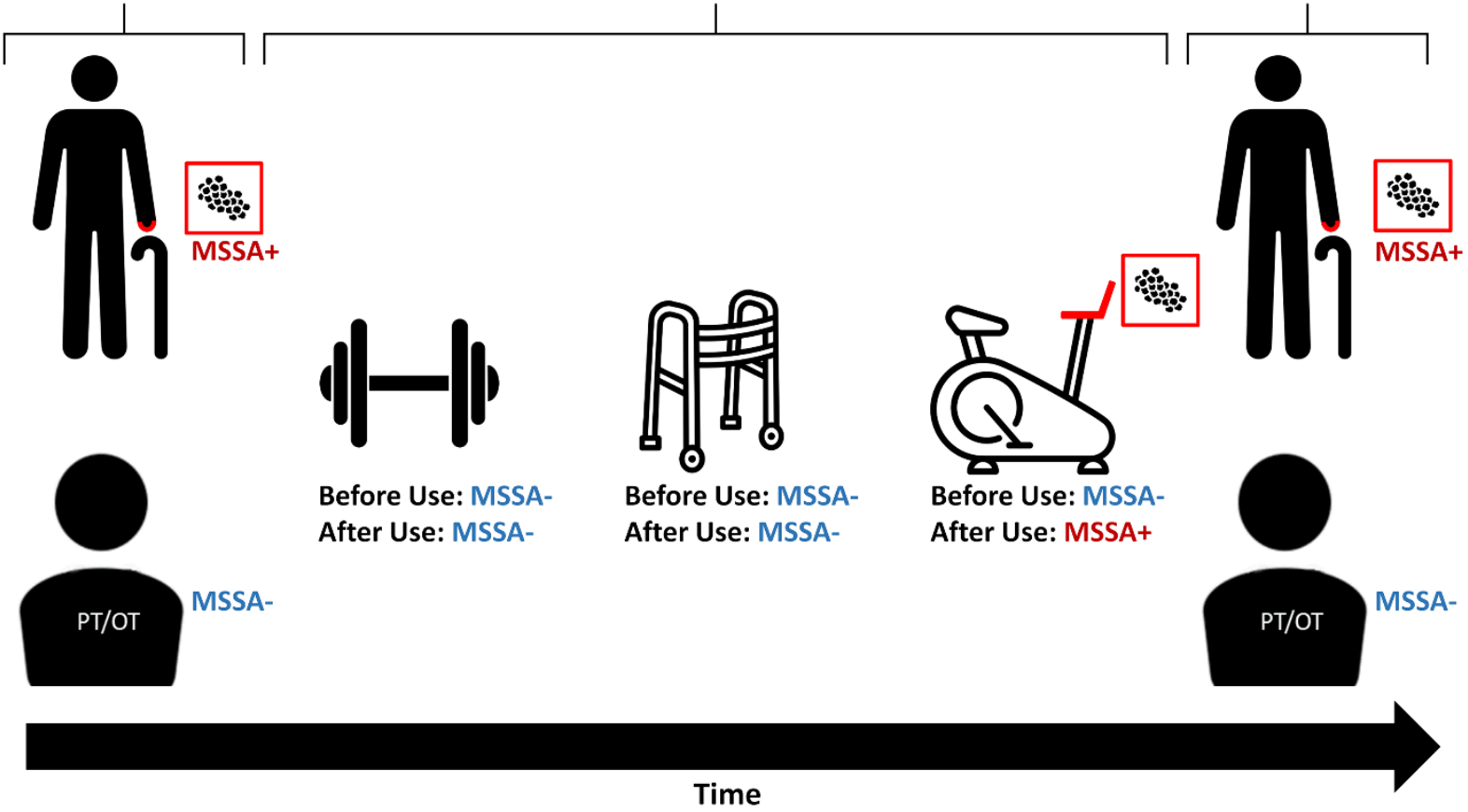

Step 3

(After Rehab Visit)

This article is protected by copyright. All rights reserved. 


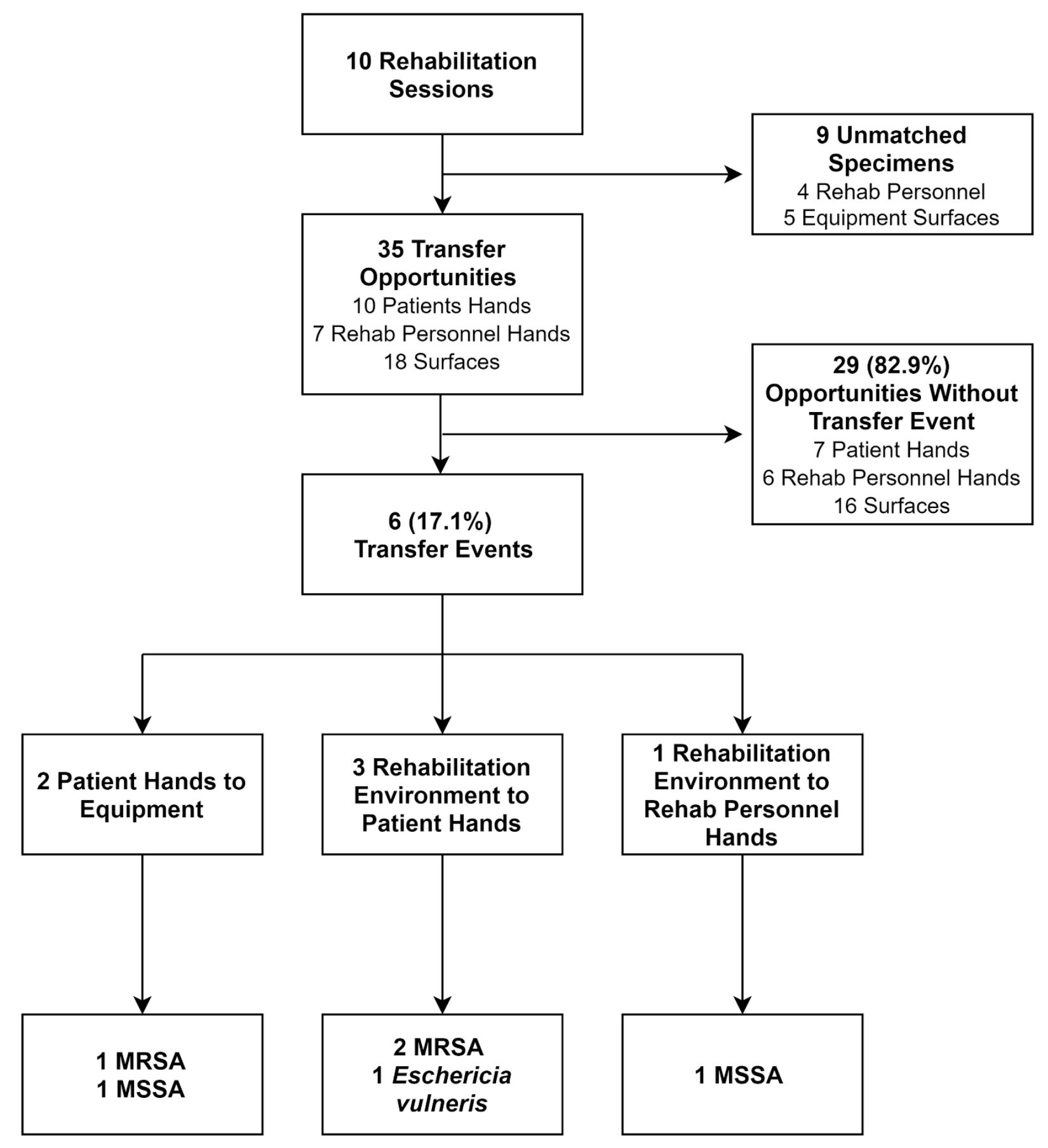

This article is protected by copyright. All rights reserved. 
SOURCE

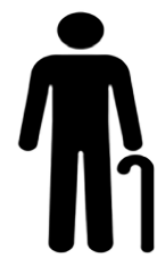

Patient Hands

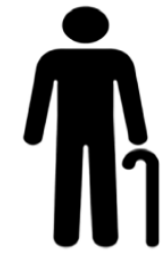

Patient Hands

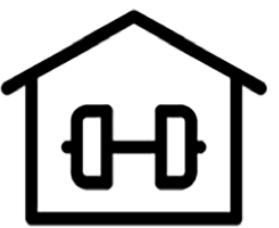

Rehab Gym

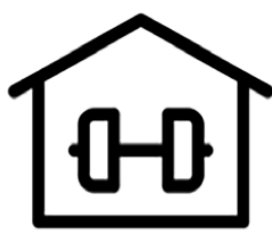

Rehab Gym

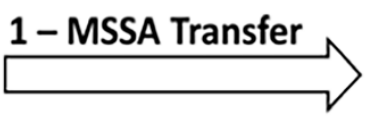

1- MRSA Transfer

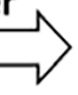

2 - MRSA Transfer

1-GNB Transfer

1 - MSSA Transfer
DESTINATION SURFACE

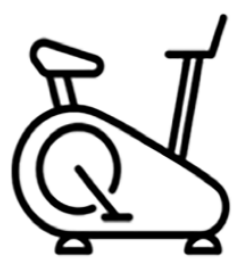

Exercise Bike

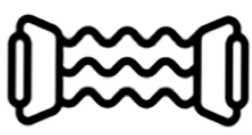

Elastic Band

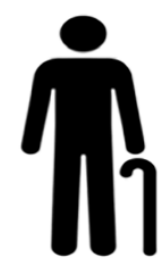

Patient Hands

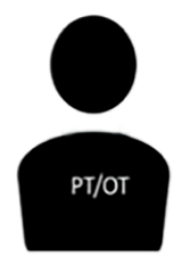

Rehab Personnel Hands 


\section{Color Charge Form}

\section{Journal of the American Geriatrics Society}

Upload this form to your submission OR return it to:

Emily Hammond / JAGS Editorial Office

jags@jjeditorial.com

\section{Dear Author:}

If you have supplied color figures in your submission, the first color figure in print is free of cost. Any additional figures you wish to print in color will incur a fee of $\$ 500$ each. Please indicate how $E A C H$ of your figures should be handled in the table below.

\begin{tabular}{|c|c|c|c|c|c|}
\hline \multirow{2}{*}{$\begin{array}{l}\text { INSTRUCTIONS: } \\
\text { For EVERY figure in your paper, } \\
\text { an X should be provided in the } \\
\text { appropriate row. }\end{array}$} & Figure No. & $\begin{array}{l}\text { Color in PRINT } \\
\text { (waive fee) }\end{array}$ & $\begin{array}{l}\text { Color in PRINT } \\
\text { (charge } \$ 500 \text { per) }^{\star}\end{array}$ & $\begin{array}{l}\text { Color ONLINE ONLY } \\
\text { (no charge) }\end{array}$ & $\begin{array}{l}\text { Black/White ONLINE } \\
\text { and PRINT }\end{array}$ \\
\hline & 1 & & & & \\
\hline $\begin{array}{l}\text { Place an ' } \mathbf{X} \text { ' in column } \mathbf{1} \text { for a } \\
\text { maximum of ONE figure. }\end{array}$ & 3 & & & & \\
\hline \multirow{2}{*}{$\begin{array}{l}\text { Place an ' } \mathbf{X} \text { ' in column } 2 \text { if you } \\
\text { want to pay for additional color } \\
\text { printing. The total in this column } \\
\text { should be entered into the } \\
\text { Quantity field below. }\end{array}$} & 5 & & & & \\
\hline & TOTAL: & & & & \\
\hline
\end{tabular}

*If you indicate ANY figures in this column, the contact information below MUST be provided.

Please Complete \& Please Print

\begin{tabular}{|c|c|c|c|}
\hline Component(s) in Article & Charge(s) & Quantity & Estimated Charge(s) (in USD) \\
\hline Color Figure Charge Standard & $\$ 500$ per figure & & ( \\
\hline & & & $=$ Total Estimated \\
\hline
\end{tabular}

\begin{tabular}{|l|}
\hline Name: \\
\hline Manuscript No:: \\
\hline Manuscript Title: \\
\hline Address: \\
\hline Phone Number: \\
\hline Fax Number: \\
\hline Email Address: \\
\hline
\end{tabular}

Verify your agreement to pay by signing and dating below. Wiley will send an invoice to the email address you've provided:

Signature: Date:

This article is protected by copyright. All rights reserved. 


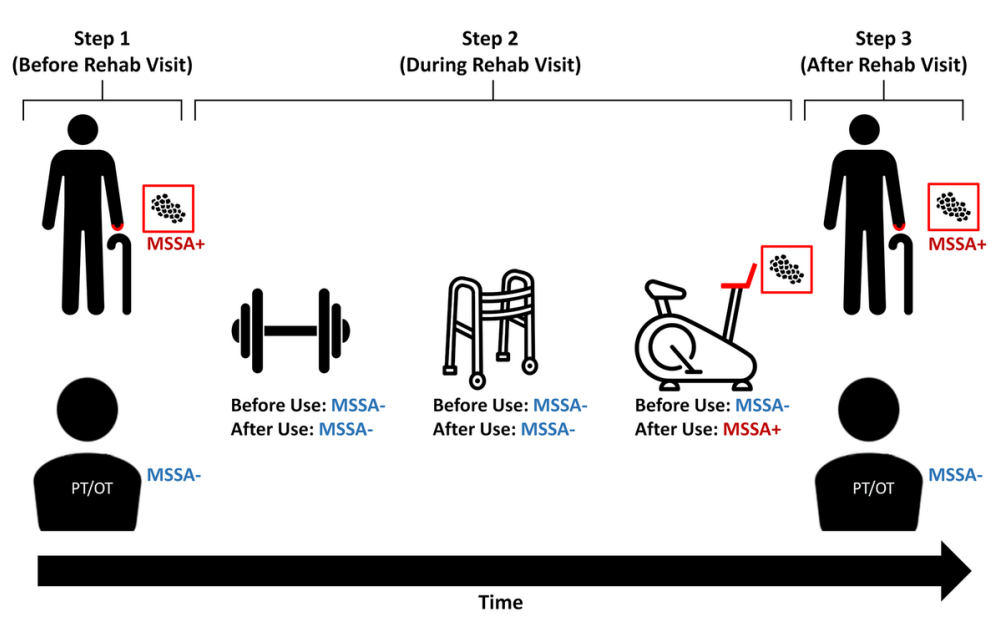

JGS_16284_JAGS Common Area Figure 1.tif 


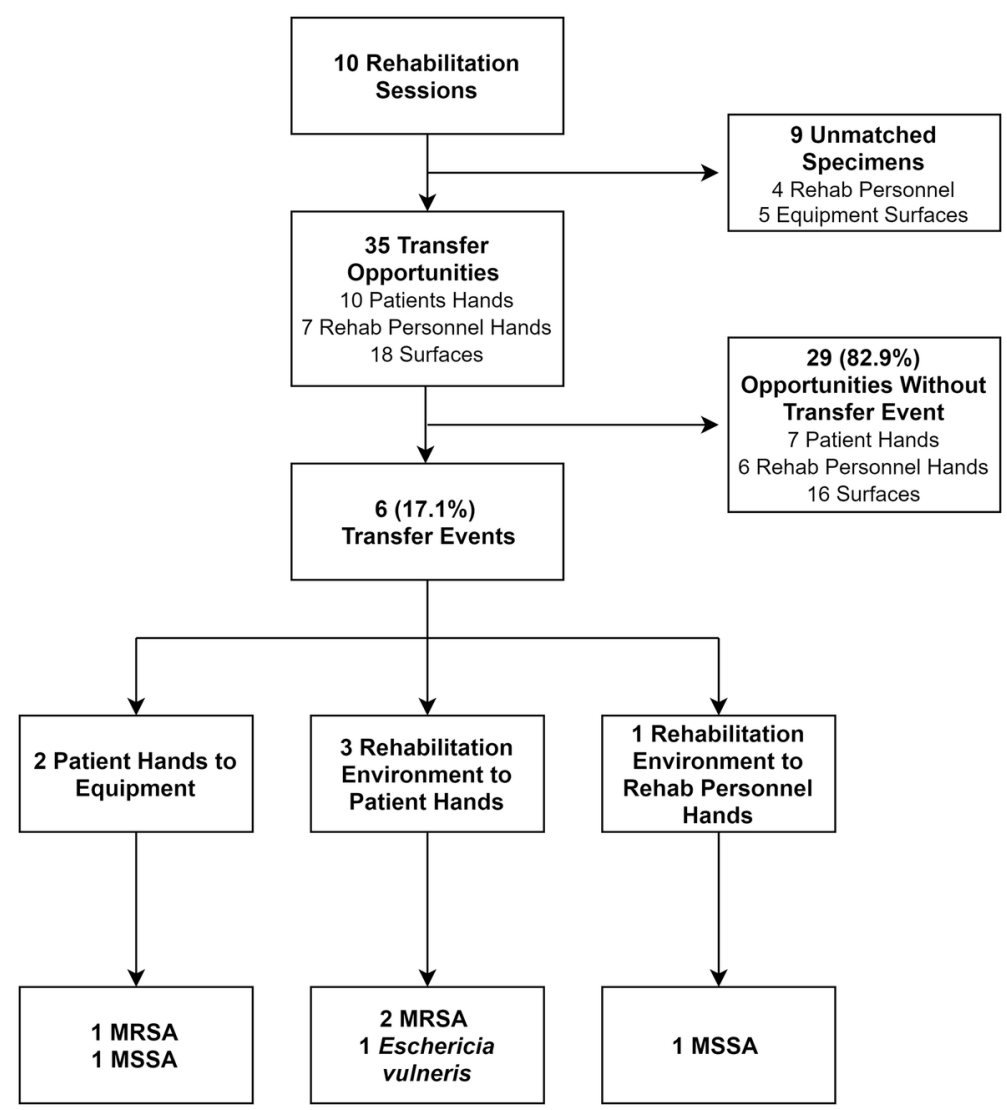

JGS_16284_JAGS Common Area Figure 2.tif

This article is protected by copyright. All rights reserved. 
SOURCE
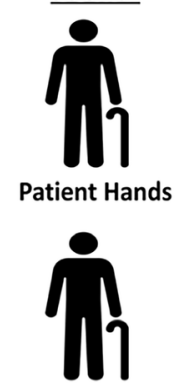

Patient Hands

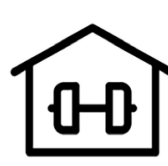

Rehab Gym

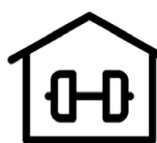

Rehab Gym
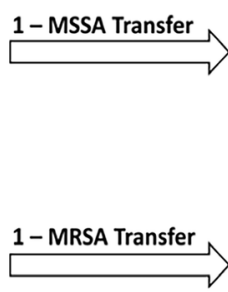

2-MRSA Transfer

$$
\text { 1-GNB Transfer }
$$

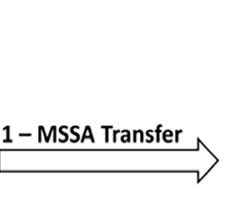

DESTINATION SURFACE
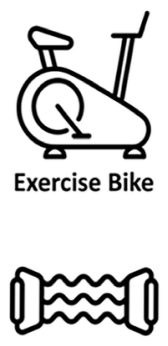

Elastic Band

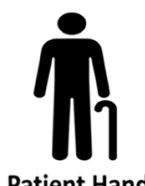

Patient Hands

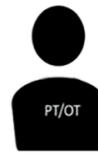

Rehab Personnel Hands

JGS_16284_JAGS Common Area Figure 3.tif 